\title{
On the Range of a Fleet of Aircraft
}

\section{A. J. Goldman}

(June 21, 1961)

\begin{abstract}
J. N. Franklin has determined the maximum range of the last member of a fleet of $n$ aircraft with identical fuel capacities and gallons-per-mile fuel efficiencies, on the assumptions that in-air exchange of fuel is unrestricted and that aircraft can be abandoned from the fleet at any stage. The present paper extends the solution (a) to the case of aircraft with equal fuel efficiencies but different fuel capacities, and (b) to the case of aircraft with equal fuel capacities but different fuel efficiencies.
\end{abstract}

\section{Introduction}

In a previous paper, ${ }^{*}$ J. N. Franklin considers the problem of determining the maximum range attainable by a fleet of $n$ aircraft with an initial total supply of $g$ gallons of fuel. It is assumed that the $i$ th aircraft has a fuel capacity of $g_{i}$ gallons and a fuel efficiency of $r_{i}$ gallons per mile. The aircraft may share fuel in flight, and any of them may be abandoned at any stage.

Franklin gives an explicit solution for $n=2$, which illustrates a dynamic programing method available for larger $n$ and also indicates the complexity of the solution in the general case. He also gives a complete discussion (including an asymptotic formula for $g$ as a function of the maximum range) of the symmetric case in which

$$
g_{i}=G, \quad r_{i}=R \quad \text { for } i=1,2, \ldots, n .
$$

In the present paper, Franklin's solution for the symmetric case is extended (a) to the situation in which the aircraft of the fleet all have the same fuel efficiency, though their fuel capacities may differ, and (b) to the case of equal fuel capacities with (possibly) different fuel efficiencies.

\section{Equal Fuel Efficiencies}

In this section it will be assumed that

$$
r_{i}=R \quad \text { for } i=1,2, \ldots, n
$$

and that the aircraft are so numbered that

$$
0<g_{1} \leq g_{2} \ldots \leq g_{n-1} \leq g_{n} .
$$

It is convenient to define

$G_{0}=0 ; G_{k}=\sum_{j=1}^{k} g_{n+1-j} \quad$ for $k=1,2, \ldots, n ; G_{n+1}=\infty$.

*J. N. Franklin, The range of a fleet of aircraft, J. Soc. Ind. Appl. Math. 8
The maximum range will be denoted by $M_{n}(g)$, or more fully by

$$
M\left(g ; g_{1}, \ldots, g_{n}\right)=M_{n}(g) .
$$

It will be shown that, if $k$ is the unique integer defined by

$$
G_{k}<g \leq G_{k+1},
$$

then the maximum range is given by

$$
\begin{array}{r}
M_{n}(g)=R^{-1}\left\{\frac{g}{k+1}+\frac{G_{1}}{1 \cdot 2}+\frac{G_{2}}{2 \cdot 3}+\cdots+\frac{G_{k}}{k(k+1)}\right\} \\
\text { if } g \leq G_{n}, \\
M_{n}(g)=R^{-1}\left\{\frac{G_{n}}{n}+\frac{G_{1}}{1 \cdot 2}+\frac{G_{2}}{2 \cdot 3}+\cdots+\frac{G_{n-1}}{(n-1) n}\right\} \\
\text { if } g>G_{n} .
\end{array}
$$

In the optimal policy, if $g \leq G_{n}$, then the flight is begun with aircraft $n, n-1, \ldots, n-k$. If $g>G_{n}$, then $g-G_{n}$ gallons of fuel must be discarded and the flight is begun with all $n$ aircraft. In any case, if the flight is begun with aircraft $n, n-1$, . . ., $n-t$, then the last-listed of these aircraft is abandoned when only $G_{t}$ gallons of fuel are left, the next-to-last is abandoned when only $G_{t-1}$ gallons remain, etc.

This result will be proved by induction on $n$. If $n=1$, then formulas (6) and (7) become

$$
M_{1}(g)=\min \left(g / R, g_{1} / R\right),
$$

which is obviously true. Now assume the result holds for all fleets of $n-1$ aircraft, where $n>1$. Let $x>0$ be the distance at which the first abandonment of an aircraft occurs. (Setting $x=0$ indicates that one or more aircraft are left at the base.) Then

$M_{n}(g)=\max _{x \leq g / n R}\left\{x+\max _{1 \leq j \leq n} M(g-n R x ;\right.$

$$
\left.\left.g_{1}, \ldots, \hat{g}_{j}, \ldots, g_{n}\right)\right\}
$$


for $g \leq G_{n}$, where the caret denotes an omitted argument. The inductive hypothesis shows that

$$
M\left(y ; y_{1}, y_{2}, \ldots, y_{n-1}\right)
$$

is a non-decreasing function of its last $n-1$ arguments over the set

$$
0 \leq y_{1} \leq y_{2} \leq \ldots \leq y_{n-1},
$$

and so, using (2), (8) becomes

$$
M_{n}(g)=\max _{x \leq g / n R}\left\{x+M\left(g-n R x ; g_{2}, \ldots, g_{n}\right)\right\}
$$

for $g \leq G_{n}$. The inductive hypothesis shows that the polygonal function

$$
P(x)=x+M\left(g-n R x ; g_{2}, \ldots, g_{n}\right)
$$

of $x$, at all points in $0<g-n R x<G_{n-1}$ for which it has a derivative, has a negative derivative, and so $P(x)$ is decreasing for $0<g-n R x<G_{n-1}$.

If $g \leq G_{n-1}$ then the maximum of $P(x)$ required in (9) is therefore obtained by setting $x=0$, yielding

$$
M_{n}(g)=M\left(g ; g_{2}, \ldots, g_{n}\right) .
$$

But by the inductive hypothesis, the right side of (11) is given by the right side of (6) for $g \leq G_{n-1}$. Thus (6) is proved for $g \leq G_{n-1}$.

Next suppose that $G_{n-1}<g \leq G_{n}$. The polygonal function $P(x)$ is decreasing for $0 \leq g-n R x \leq G_{n-1}$, as noted above, and is increasing for $g-n \bar{R} x>G_{n-1}$, since by (7) and the inductive hypothesis the second summand in (10) is constant in this range. Thus the maximum of $P(x)$ required in (9) is obtained by setting $g-n R x=G_{n-1}$, and (9) becomes

$$
\begin{gathered}
M_{n}(g)=\frac{g-G_{n-1}}{n R}+M\left(G_{n-1} ; g_{2}, \ldots, g_{n}\right)=\frac{g-G_{n-1}}{n R} \\
+R^{-1}\left\{\frac{G_{n-1}}{n-1}+\frac{G_{1}}{1 \cdot 2}+\ldots+\frac{G_{n-2}}{(n-2)(n-1)}\right\} \\
=R^{-1}\left\{\frac{g}{n}+\frac{G_{1}}{1 \cdot 2}+\ldots \frac{G_{n-1}}{(n-1) n}\right\},
\end{gathered}
$$

so that (6) is proved for $G_{n-1}<g \leq G_{n}$. Finally, (7) follows from (6) and the obvious fact that

$$
M_{n}(g)=M_{n}\left(G_{n}\right) \quad \text { if } g>G_{n} .
$$

\section{Equal Fuel Capacities}

In this section it will be assumed that

$$
g_{i}=G \quad \text { for } i=1,2, \ldots, n
$$

and that the aircraft are so numbered that

$$
r_{1} \geq r_{2} \ldots \geq r_{n-1} \geq r_{n}>0 .
$$

It is convenient to define

$$
R_{k}=1 / \sum_{j=1}^{k} r_{n+1-j} \quad \text { for } k=1,2, \ldots, n .
$$

The maximum range is denoted $M_{n}(g)$, or more fully by

$$
M\left(g ; r_{1}, \ldots, r_{n}\right)=M_{n}(g) .
$$

If $g \leq n G$, then there is a unique integer $k$ for which

$$
k G<g \leq(k+1) G .
$$

The maximum range is given by

$$
\begin{aligned}
M_{n}(g)=G\left(R_{1}+R_{2}+\ldots+R_{k}\right)+R_{k+1}(g-k G) & \\
& \text { if } g \leq n G, \\
M_{n}(g)=G\left(R_{1}+R_{2}+\ldots+R_{n}\right) & \text { if } g>n G .
\end{aligned}
$$

In the optimal policy, if $g \leq n G$, the flight is begun with aircraft $n, n-1, \ldots, n-k$. If $g>n G$, then $g-n G$ gallons of fuel must be discarded and the flight is begun with all $n$ aircraft. In any case, if the flight is begun with aircraft $n, n-1$, . . , $n-t$, then the last-listed of these aircraft is abandoned when only $t G$ gallons of fuel are left, the next-to-last is abandoned when only $(t-1) G$ gallons remain, etc.

The proof of this result is just like that in the preceding section, and is therefore omitted. By way of orientation, it may be noted that the analog of (9) is

$$
M_{n}(g)=\max _{x \leq R_{n} g}\left\{x+M\left(g-R_{n}^{-1} x ; r_{2}, \ldots, r_{n}\right)\right\} .
$$

(Paper 65B4-61) 